\title{
État des lieux des infections associées aux soins dans deux hôpitaux publics du sud Benin (Afrique de l'ouest) : Centre Hospitalier Universitaire de Zone d'Abomey- Calavi/Sô-Ava et Centre Hospitalier de Zone de Cotonou 5.
}

\author{
F Cyr Doscoph AFLE*1, Kisito J.M.K. QUENUM², Septime HESSOU1, Roch Christian JOHNSON1. \\ 1. Centre Interfacultaire de Formation et de Recherche en Environnement pour le Développement (CIFRED), Université \\ d'Abomey-Calavi (UAC). E-mail : cyrafle@yahoo.fr Téléphone: 00229 66043704, Roch Christian JOHNSON1 : \\ rochchristianjohnson@gmail.com Téléphone : 0022996221132
}

2. Faculté de Médecine, Université de Parakou (Benin). E-mail : kisitog@gmail.com Téléphone : 0022994512122

*Corresponding author Mail : cyrafle@yahoo.fr

Original submitted in on $4^{\text {th }}$ November 2017. Published online at www.m.elewa.org on $31^{\text {st }}$ January 2018 https://dx.doi.org/10.4314/iab.v121i1.9

\section{RESUME}

Objectif : Le présent travail a pour objectif de déterminer la prévalence et les facteurs liés aux infections associées aux soins dans le Centre Hospitalier et Universitaire de Zone d'Abomey-Calavi/So-Ava et le Centre Hospitalier de Zone de Cotonou 5 au sud Benin (Afrique de l'ouest).

Méthodologie et résultats: L'étude a été réalisée en avril 2017 dans deux hôpitaux du sud Bénin. Le diagnostic des infections est conforme aux définitions internationales des Centers for Disease Control and Prevention (CDC). Ce diagnostic est également cohérent avec les critères internationaux de la définition des infections associées aux soins et les actualisations du Comité Technique des Infections Nosocomiales et des Infections Liées aux Soins (CTINILS) France. La prévalence d'infections associées aux soins est de 14,39 \% pour 139 patients inclus dans l'étude. Le sondage vésical en est un facteur de risque $(O R=2,45 ; C h i-2=42,81$ et $p<10$ 3 ). Des bactéries résistantes à la méthicilline, et aux céphalosporines de troisième génération ont été identifiées.

Conclusion et application des résultats : L'étude rapporte la prévalence des infections associées aux soins et révèle l'existence de bactéries multirésistantes dans le Centre Hospitalier Universitaire de Zone d'AbomeyCalavi/Sô-Ava et le Centre Hospitalier de Zone de Cotonou 5 au Bénin. Elle met en évidence l'ampleur du problème de santé, témoin de la qualité des soins dans ces hôpitaux. C'est un indicateur de sensibilisation du personnel soignant à un programme opérationnel rigoureux en hygiène hospitalière. II y a également une nécessité de maîtriser les résistances antimicrobiennes. Ainsi, nous devons déconseiller l'automédication et recommander une antibiothérapie efficace.

Mots clés : Infections associées aux soins, Prévalence, résistance antimicrobienne, contrôle de l'infection, surveillance, Bénin. 

sud Benin: Centre Hospitalier Universitaire de Zone d'Abomey-Calavi/Sô-Ava et de Zone de Cotonou 5.

\section{ABSTRACT}

Objective : The aim of this work is to determine the prevalence and factors of healthcare-associated infections in the University Hospital of Abomey-Calavi / So-Ava and Cotonou 5 Hospital in South Benin (West Africa). Methodology and results: The study was conducted in April 2017 in two hospitals in South Benin. The diagnosis of the infections is in compliance with the international definitions of Centers for Disease Control and Prevention (CDC). This diagnosis is also consistent with the international criteria for the definition of healthcare-associated infections and updates of CTINILS France. Of 139 patients included in the study, there is an overall prevalence of infected patients of $14.39 \%$. Urinary catheter is a risk factor of urinary tract infection (OR= 2.45; $x 2=$ 42.81 and $p<10-3)$. Resistance of leading pathogens identified to antimicrobials include methicillin-resistance and to third-generation cephalosporins.

Conclusion and application of results : The study reports the prevalence of healthcare-associated infections and reveals the existence of multidrug-resistant bacteria in University Hospital of Abomey-Calavi / So-Ava and Cotonou 5 Hospital in South Benin (West Africa). It highlights the extent of the health problem, witnessing the quality of care in these hospitals. It is an indicator of awareness of the nursing staff for a rigorous operational program in hospital hygiene. There is also a need to control antimicrobial resistance. Thus, we must advise against self-medication and recommend effective antibiotic therapy.

Keywords: Healthcare-associated infections, Prevalence, Antimicrobial resistance, Infection control, Surveillance, Benin.

\section{INTRODUCTION}

Les infections associées aux soins (IAS) constituent un problème de santé qui survient au cours ou au décours d'une prise en charge d'un patient (Parneix, 2010). L'appellation « infections associées aux soins » introduite en 2007 en France par le Comité Technique des Infections Nosocomiales et des Infections Liées aux Soins (CTINILS) est une adaptation à l'évolution internationale des concepts (Parneix, 2010). A cet effet, le CTINILS donne à l'expression «infection nosocomiale » qui a une connotation jugée souvent péjorative malgré son ancrage dans divers textes réglementaires, le sens d'une infection associée aux soins contractée dans un établissement de santé (Parneix, 2010). La survenue de ces infections est favorisée par de nombreux microorganismes appartenant à la flore hospitalière (Hamza, 2010) avec une prépondérance des staphylocoques et des bacilles à Gram négatif (Soussy, 2010, Bagheri Nejad et al., 2011 ; Pividori et Le Heurt, 2011). Ces germes présentent parfois des résistances aux antibiotiques (Goettsch et al., 2000, Daza et al., 2001) et l'on enregistre ainsi une forte prévalence de Staphylococcus aureus résistant à la méthicilline (SARM) (Pourakbari et al., 2011), des Enterococcus résistant à la vancomycine (ERV) (Pourakbari et al., 2012), et de Pseudomonas aeruginosa multirésistantes (Movahedi et al., 2013).
Dès lors, cette résistance aux antimicrobiens qui survient dans la lutte contre ce problème de santé, peut incontestablement constituer une réelle difficulté pour la réalisation des objectifs de développement durable notamment l'objectif de développement durable 3 (ODD 3) (OMS 2016). En dehors des souches bactériennes souvent identifiées lors de ces infections hospitalières, de nombreux virus appartenant à des familles virales distinctes dont les Retroviridae et les virus des hépatites sont aussi responsables des IAS (Agut, 2010). Par ailleurs, il existe également d'autres pathogènes particuliers que sont les prions encore appelés agents transmissibles non conventionnels (ATNC) responsables de certaines IAS (encéphalopathies spongiformes) (Agut, 2010). La survenue des infections associées aux soins en milieux hospitalier est également favorisée par de nombreux facteurs dont les facteurs liés aux malades, les expositions aux dispositifs médicaux invasifs et les insuffisances dans la qualité des soins (Durocher, 2005; Espinasse et al., 2010). II en est de même des défauts de nettoyage, de désinfection et de stérilisation qui sont aussi des facteurs potentiels d'infection associée aux soins (Sydnor et Perl, 2011 ; Ghazi et al., 2012; Abreu et al., 2013). Les infections contractées en milieu hospitalier ont des 

sud Benin: Centre Hospitalier Universitaire de Zone d'Abomey-Calavi/Sô-Ava et de Zone de Cotonou 5.

conséquences variée, dont d'une augmentation de la morbidité et de la mortalité hospitalière, surtout dans les pays en développement (Hamza, 2010). Ainsi, dans les hôpitaux de soins aigus, les infections associées aux soins sont la cinquième cause de décès (Sydnor et Perl, 2011). Dans le monde, plus de 1,4 million de personnes souffrent d'infections contractées à l'hôpital, et le risque d'en contracter est deux (02) à vingt (20) fois plus élevé dans les pays en développement que dans les pays développés (OMS, 2017). La proportion de patients infectés lors des soins de santé dans certains pays en développement peut dépasser $25 \%$ (OMS, 2017). En Afrique, la prévalence des infections associées aux soins varie entre $2,5 \%$ et $14,8 \%$ (Bagheri Nejad et al., 2011). Au Bénin, la prévalence enregistrée chez les patients hospitalisés au Centre National Hospitalier et Universitaire Hubert Koutoukou Maga (CNHU-HKM) en 2012 était de $9,84 \%$ (Ouendo et al., 2015). Au plan national, dans $87 \%$ des hôpitaux inclus dans une enquête, elle était de 19,1 \% (Ahoyo et al., 2014). Les infections associées aux soins sont ainsi à l'instar d'autres pays, un problème de santé publique au Bénin. Le phénomène infectieux peut rester à l'échelon individuel, mais il peut malheureusement toucher plusieurs personnes malades dans une même unité de soins, et dans des cas extrêmes inciter à fermer cette unité. La survenue de ce problème de santé au cours ou au décours d'un soin dans un établissement de santé pose un problème éthique dans un contexte où c'est pendant l'hospitalisation du patient initialement indemne, que l'infection est contractée et devient sa préoccupation additionnelle. Le retentissement de ce problème additionnel affecte l'entourage du patient et engendre un coût social et familial (maison de rééducation, non-reprise de travail, perte d'emploi, dépression) (Le Heurt et al., 2002 ; Pividori et Le Heurt, 2011). Dans certains cas, une prolongation de l'hospitalisation du patient évaluée entre 9 et 11 jours en moyenne est nécessaire pour la prise en charge de l'infecction (Stone et al., 2005). L'enjeu économique qui en découle est le surcoût financier important pour les ménages et le système de santé (Haley et al., 1985 ; Pittet et al., 1999). Face à ce problème de santé publique, au Bénin, les méthodes de la lutte contre les infections contractées dans les établissements de santé sont inscrites dans la politique nationale d'hygiène hospitalière (Décret $\mathrm{N}^{\circ}$ 2006-087). Cette politique inscrit la surveillance et la recherche sur les IAS dans les objectifs des établissements de santé. Très souvent, cependant, l'insuffisance des ressources dans les pays en développement, rendent très élémentaires les actions de lutte contre les infections acquises à l'hôpital malgré l'existence des directives dans le domaine (Simon et al., 2007 ; Allegranzi et al., 2011). Ainsi, les infections associées aux soins sont constamment sousestimées et mal connues car elles sont peu surveillées. Au Bénin, cela se traduit par l'absence de données épidémiologiques liées aux IAS dans les annuaires statistiques sanitaires traduit les limites et les insuffisances de la surveillance de ce problème de santé. Mais lorsque la surveillance est bien réalisée, la quantification des IAS qui en résulte permet d'évaluation l'ampleur du problème, et de justifier les ressources affectées aux différentes activités, une étape de la prévention (NNIS, 2004; Coignard et al., 2007). L'objectif de cette étude conduite en Avril 2017 a été de déterminer la prévalence et les facteurs associés aux IAS dans le Centre Hospitalier Universitaire de Zone d'AbomeyCalavi/So-Ava (CHUZ Abomey-Calavi/So-Ava) et le Centre Hospitalier de Zone de Cotonou 5 (CHZ Cotonou 5). Les données épidémiologiques sur les IAS ainsi générées du travail dans ces deux centres hospitaliers suivies d'un retour d'information aux services, sensibilisent les professionnels de santé sur la nécessité de l'observance des règles d'hygiène dans la lutte contre ces infections. Ces règles d'hygiène hospitalière sont beaucoup plus des mesures de prévention souvent moins onéreuses par rapport au coût et exigences de la prise en charge d'une infection déclarée et documentée dans les pays en développement à ressources limitées. 

sud Benin: Centre Hospitalier Universitaire de Zone d'Abomey-Calavi/Sô-Ava et de Zone de Cotonou 5.

\section{MATERIELS ET METHODES}

Les patients inclus dans cette étude, ont été les présents dans le CHUZ Abomey-Calavi/So-Ava et le CHZ Cotonou 5 pendant le déroulement de l'enquête. Le choix des services a été fait sur la base du niveau de risque d'infection. Les infections associées aux soins enregistrées ont été fonction de l'unité de soins ; du recrutement des patients dans le service, des thérapeutiques (actes invasifs) (Hamza, 2010). Les services de la médecine, la maternité, la pédiatrie, l'hospitalisation chirurgicale ont été sélectionnés dans le CHUZ Abomey-Calavi/So-Ava. Les services de pédiatrie, maternité, réanimation et hospitalisation adulte ont été retenus dans le Centre Hospitalier de Zone de Cotonou 5. Ainsi, cette étude a porté sur 139 patients hospitalisés et qui y ont séjourné pendant au moins 48 heures à la date de l'enquête. Le CHUZ Abomey-Calavi/So-Ava a enregistré 71 patients, et le $\mathrm{CHZ}$ Cotonou 5 ; 68 patients. Les variables d'étude ont été:

- les caractéristiques sociodémographiques du patient : âge, sexe, durée d'hospitalisation ;

- les variables cliniques et paracliniques liées aux infections associées aux soins ;

- les variables associées aux soins et actes invasifs: sonde urinaire, cathéter vasculaire central, cathéter vasculaire périphérique, alimentation parentérale, ventilation mécanique, intervention chirurgicale dans les 30 jours précédant le jour d'enquête et 12 mois en cas de prothèse chirurgicale. Le recueil d'information a été fait sur la base du dépouillement des dossiers médicaux des patients, ceux des soins infirmiers et les résultats de laboratoire, en plus de l'interrogatoire

\section{RESULTATS}

Population étudiée : L'enquête a porté sur 139 patients. L'âge minimum est de un (01) an, et le maximum 72 ans. La médiane est de 30 ans et l'étendue inter-quartiles est de 12 ans à 42 ans. Dans la population d'étude, la durée d'hospitalisation varie de deux (02) jours à 24 jours avec une médiane qui est de cinq (05) jours. La durée minimum d'hospitalisation de deux (02) jours est identique aux deux établissements de santé. La plus longue durée de 24 jours d'hospitalisation est enregistrée dans le CHUZ d'Abomey-Calavi/So-Ava, contre 14 jours dans l'hôpital de zone de Cotonou 5. A partir des durées d'hospitalisation ainsi considérées dans la population de du personnel. Sur le plan opérationnel, nous avons retenu les critères de Centers for Disease Control and Prevention (CDC, 2017). Ces critères définissent le sepsis à partir des différents paramètres vitaux: le nombre de battements cardiaques par minute, la température corporelle, la fréquence respiratoire, et le nombre de globules blancs. Les critères internationaux de la définition des infections associées aux soins contenus dans le document des 100 recommandations proposées par le comité technique national des infections nosocomiales en France et de leur actualisation ont également permis le diagnostic des infections associées aux soins (CTINILS, 2007). Ces recommandations identifient les spécificités sémiologiques de chaque type d'infection associée aux soins (IAS). Les informations relatives aux patients du même service ont été recueillies le même jour dans chaque hôpital par des infirmiers formés à cet effet. Un correspondant médical (médecin) externe au service d'enquête a confirmé les diagnostics d'infections associées aux soins colligées.

Traitement et analyse des données : Les données ont été saisies et analysées à l'aide du logiciel statistical package for social sciences 21 (SPSS). Le test de Fisher (F) a été utilisé pour tester l'indépendance entre services et présence d'infection associée aux soins chez les patients. Le test de Chi-2 de Pearson a été utilisé pour tester l'indépendance entre facteurs associées et la présence d'infection associée aux soins chez les patients. Un odds ratio $(O R)$ a été calculé pour mesurer l'association entre l'exposition à un facteur et l'infection associée aux soins.

139 patients, l'étendue inter-quartiles est de deux (02) jours à huit (08) jours d'hospitalisation.

Prévalence des infections associées aux soins: Parmi les 139 patients inclus dans l'étude, 20 ont une infection associée aux soins, soit une prévalence de $14,39 \%$. Le Centre Hospitalier et Universitaire de Zone d'Abomey-Calavi/So-Ava présente neuf (09) cas d'IAS sur 71 patients enregistrés avec une prévalence de 12,68 $\%$. Le Centre Hospitalier de Zone de Cotonou 5 enregistre 11 cas d'IAS sur 68 patients du centre inclus dans l'étude avec une prévalence de 16,18\%. Cette prévalence des infections associées aux soins par service et par centre hospitalier se présente dans le tableau 1. 
Tableau1 : Prévalence des infections associées aux soins dans la population des 139 patients ( 68 pour $\mathrm{CHZ}$ de Cotonou 5, et 71 pour le CHUZ Abomey-Calavi/Sô-Ava).

\begin{tabular}{l|l|c|l|r}
\hline \multirow{2}{*}{ Centre hospitalier } & \multicolumn{2}{|c|}{ CHUZ Abomey-Calavi/ Sô-Ava } & \multicolumn{2}{c}{ CHZ Cotonou5 } \\
\cline { 2 - 5 } Spécialité du service & IAS & $\%$ & IAS & - \\
\hline Médecine & 2 & 02,82 & - & - \\
Hospitalisation chirurgie & 2 & 02,82 & - & 0 \\
Maternité & 3 & 04,23 & 0 & 11,76 \\
Hospitalisation Adultes & - & - & 8 & 01,47 \\
Réanimation & - & - & 1 & 02,94 \\
Pédiatrie & 2 & 02,82 & 2 & 16,18 \\
Total & 9 & 12,68 & 11 & \\
\hline
\end{tabular}

Parmi les services du CHUZ Abomey-Calavi/So-Ava, la maternité enregistre la plus grande prévalence en infections associées aux soins $(4,23 \%)$ (tableau1). Les autres services ont enregistré de différentes proportions. Dans le CHUZ Abomey-Calavi/So-Ava, II n'y a pas une association entre les différentes prévalences et les services $(F=1,52 ; p=0,80)$. Au centre Hospitalier de Zone de Cotonou 5 , le service hospitalisation adultes enregistre une prévalence prépondérante de $11,76 \%$ d'IAS. II n'y a pas une association entre les services et les prévalences enregistrées $(F=1,44 ; p=0,75)$.
Sites de localisation des infections associées aux soins : Dans l'ensemble des 20 infections associées aux soins enregistrées, les infections respiratoires basses et les infections du site opératoire sont les plus nombreuses (tableau 2). Les infections du site opératoire sont en plus grande proportion $(7,04 \%)$ au CHUZ Abomey Calavi/Sô-Ava. Dans le CHZ Cotonou 5, ce sont les infections respiratoires basses sont les plus fréquentes $(7,35 \%)$.

Tableau 2 : fréquences de la localisation des différents sites d'infections associées aux soins dans la population des 139 patients (68 pour CHZ de Cotonou 5, et 71 pour le CHUZ Abomey-Calavi/Sô-Ava).

\begin{tabular}{|c|c|c|c|c|c|}
\hline & \multicolumn{2}{|c|}{$\begin{array}{l}\text { CHUZ Abomey-Calavi/ Sô-Ava } \\
(\mathrm{N}=71)\end{array}$} & \multicolumn{2}{|c|}{$\begin{array}{l}\text { CHZ Cotonou } 5 \\
\quad(N=68)\end{array}$} & $\begin{array}{c}\text { Ensemble des deux centres } \\
\text { hospitaliers } \\
(\mathrm{N}=139)\end{array}$ \\
\hline & Nombre & Fréquence $(\%)$ & Nombre & Fréquence (\%) & Nombre \\
\hline IU & 1 & 1,41 & 3 & 4,41 & 4 \\
\hline $\mathbb{R}$ & 3 & 4,23 & 5 & 7,35 & 8 \\
\hline ISO & 5 & 7,04 & 3 & 4,41 & 8 \\
\hline Autres & 0 & 0 & 0 & 0 & 0 \\
\hline
\end{tabular}

IU : infections urinaires, IR : infections respiratoires basses, ISO : infections du site opératoire, Autres : les infections gastro-intestinales, les infections des os et articulations ; les infections de l'œil ; de l'oreille ; du nez ; de la gorge et de la bouche ; les infections cardio-vasculaires et les infections du système nerveux.

Micro-organismes isolés des infections associées aux soins : Parmi les 20 infections associées aux soins, $60 \%$ ont bénéficié d'une exploration microbiologique. Les infections respiratoires basses au nombre de 8, (40\%) n'ont pas fait l'objet d'un diagnostic microbiologique. Les micro-organismes identifiés (Tableau 3 ) sont les bacilles
Gram négatif (12/18), suivis des cocci Gram positifs (6/18). Staphylococcus aureus représente le tiers des germes isolés des infections associées aux soins documentées. Escherichia coli (4/18) et Pseudomonas aeruginosa (4/18) viennent en seconde place. 

sud Benin: Centre Hospitalier Universitaire de Zone d'Abomey-Calavi/Sô-Ava et de Zone de Cotonou 5.

Tableau 3 : répartition des micro-organismes identifiés dans l'ensemble des deux centres hospitaliers en fonction du site de l'infection associée aux soins

\begin{tabular}{|c|c|c|c|c|c|c|}
\hline \multirow[b]{2}{*}{ micro-organismes } & \multicolumn{2}{|c|}{$\begin{array}{l}\text { Site opératoire } \\
\qquad(n=10)\end{array}$} & \multicolumn{2}{|c|}{$\begin{array}{l}\text { Site urinaire } \\
\quad(n=8)\end{array}$} & \multicolumn{2}{|c|}{$\begin{array}{c}\text { Voies respiratoires } \\
\text { basses }(n=8)\end{array}$} \\
\hline & Nbre & $\%$ & Nbre & $\%$ & Nbre & $\%$ \\
\hline Acinetobacter spp & 0 & 0 & 2 & 25 & 0 & 0 \\
\hline Klebsiella pneumoniae & 0 & 0 & 2 & 25 & 0 & 0 \\
\hline Escherichia coli & 0 & 0 & 4 & 50 & 0 & 0 \\
\hline Pseudomonas aeruginosa & 4 & 40 & 0 & 0 & 0 & 0 \\
\hline Staphylococcus aureus & 6 & 60 & 0 & 0 & 0 & 0 \\
\hline Non documenté & 0 & 0 & 0 & 0 & 8 & 100 \\
\hline
\end{tabular}

Dans chaque centre hospitalier, 9 germes ont été identifiés, et les proportions occupées par ce total de 18 microorganismes isolés sont présentées par la figure 1 en fonction de chaque centre hospitalier.

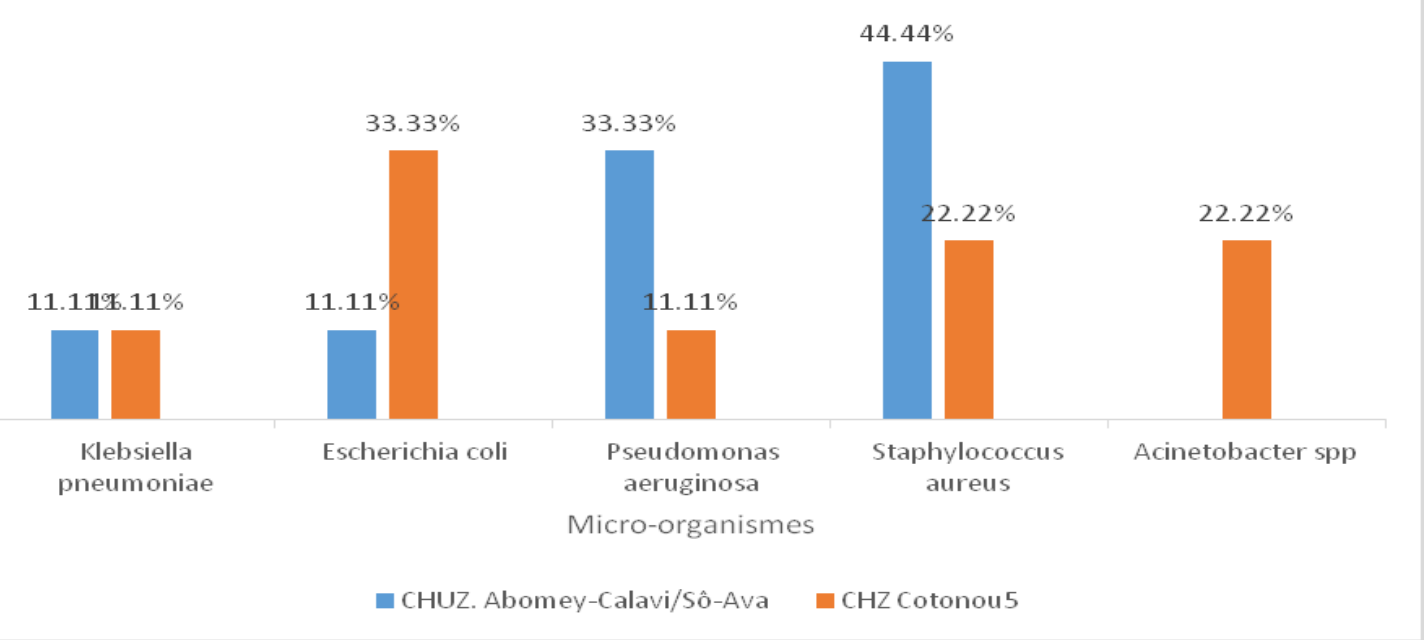

Figure 1 : répartition des micro-organismes identifiés dans les infections associées aux soins recensées au Centre Hospitalier Universitaire de Zone d'Abomey-Calavi/So-Ava et l'hôpital de Zone de Cotonou 5.

Sensibilité des bactéries aux antibiotiques : Des 18 bactéries identifiées, $8(44,44 \%)$ étaient résistantes aux antibiotiques (Tableau 4). Six (06) bactéries étaient résistantes aux Céphalosporines de Troisième
Génération (C3G); deux (02) bactéries résistantes aux quinolones; un (01) bactérie aux pénicillines ; et deux (02) bactéries à la méthicilline.

Tableau 4. Répartition des micro-organismes isolés en fonction de leurs sensibilités aux antibiotiques

\begin{tabular}{|c|c|c|c|}
\hline Bactéries & $\begin{array}{c}\text { Total des bactéries } \\
\text { identifiées }\end{array}$ & $\begin{array}{l}\text { Nombre de bactéries sensibles aux } \\
\text { antibiotiques testés (usuels) }\end{array}$ & $\begin{array}{l}\text { Nombre de bactéries } \\
\text { multi résistantes }\end{array}$ \\
\hline Acinetobacter spp & 2 & 1 & $\begin{array}{l}1 \text { aux C3G, aux } \\
\text { quinolones }\end{array}$ \\
\hline Klebsiella pneumoniae & 2 & 1 & 1 aux C3G \\
\hline Escherichia coli & 4 & 3 & $\begin{array}{c}1 \text { au C3G, quinolones } \\
\text { Pénicillines }\end{array}$ \\
\hline Pseudomonas aeruginosa & 4 & 1 & 3 aux C3G \\
\hline Staphylococcus aureus & 6 & 4 & 2 à la méthicilline \\
\hline Total & 18 & 10 & 8 \\
\hline
\end{tabular}



sud Benin: Centre Hospitalier Universitaire de Zone d'Abomey-Calavi/Sô-Ava et de Zone de Cotonou 5.

Facteurs associés aux IAS : Dans le $\mathrm{CHZ}$ Cotonou 5 , le sondage vésical a été réalisé chez 8 patients. Le sondage vésical a été réalisé chez 15 patients dans cette étude et est significativement associé à la survenue d'une IAS

\section{DISCUSSION}

Des progrès sensibles ont été accomplis dans l'accroissement de l'espérance de vie et la réduction de certaines causes majeures de la mortalité infantile et maternelle, ainsi que d'autres maladies (ONU 2015). L'apparition de la pénicilline en 1945 a montré que les grandes infections semblaient vaincues, mais parallèlement avec le développement des techniques invasives et l'insuffisance des mesures d'hygiène hospitalière dans certains cas, les infections associées aux soins sont redevenues un problème de santé publique. Cette situation accentuée avec l'émergence des bactéries pathogènes résistantes aux antibiotiques, oblige l'équipe soignante à une attitude thérapeutique plus rigoureuse dans la prise en charge des patients (OMS 2016). L'objectif de l'étude a été de déterminer la prévalence et les facteurs associés à ces infections dans le Centre Hospitalier Universitaire de Zone d'AbomeyCalavi/Sô-Ava puis le Centre Hospitalier de Zone de Cotonou 5. L'étude a porté sur 139 patients âgés d'un (01) an à 72 ans. La plus longue durée d'hospitalisation de 24 jours a été obtenue au CHUZ Abomey-Calavi/SôAva. La prévalence des IAS a été de $14,39 \%$ au sein des 139 patients inclus dans l'étude. Cette proportion est supérieure au $5,7 \%$ obtenue dans une étude réalisée en 2007 au CHU Hassan II de Fès au Maroc (El Rhazi et al., 2007). Elle est également plus élevée que celle des pays développés qui va de 5 à 10\% (Blomberg et al. 2004). En 2012, Ahoyo et ses pairs ont rapporté une prévalence de $19,1 \%$ pour 3130 patients inclus dans une étude au Bénin. Cette prévalence est supérieure au 14,39\% obtenue dans le présent travail. La différence entre la prévalence de notre étude et celle de Ahoyo et al en 2012 pourrait être due aux nombres des patients inclus dans chaque étude. Gastmeier et al (1998) ont rapporté que la prévalence des infections associées aux soins augmente avec le niveau de technicité et la taille des établissements. Mais, les résultats de notre étude présentent une situation contraire, où la prévalence des IAS est de 16,18\% dans le $\mathrm{CHZ}$ de Cotonou 5 qui est un centre non universitaire, contre $12,68 \%$ enregistrée au CHUZ Abomey-Calavi/So-Ava qui est un centre universitaire. Dans chaque centre hospitalier de l'étude, la prévalence des infections associées aux soins a varié d'un service à l'autre. Toutefois, dans le CHUZ AbomeyCalavi/So-Ava, II n'y a pas une association entre les
$\left(\mathrm{OR}=2,45 ; \quad\right.$ Chi-2 $=42,81$ et $\left.p<10^{-3}\right)$. L'intervention chirurgicale réalisée chez 47 patients de l'étude, n'est pas significativement associée à la survenue d'une IAS $(\mathrm{OR}=1,37$; Chi-2 $=0,4$ et $p=0,35)$.

différentes prévalences et les services $(F=1,52 ; p=0,80)$. II en est de même pour le $\mathrm{CHZ}$ de Cotonou 5 , sans une association entre les services et les prévalences enregistrées $(F=1,44 ; p=0,75)$. Les infections respiratoires basses $(n=8)$ et les infections du site opératoire $(n=8)$ étaient les plus fréquentes de l'ensemble IAS identifiées. Ces résultats concordent avec ceux de Bezzaoucha et al (1994), qui ont trouvé que les infections du site opératoire constituaient les infections les plus répandues, avec des fréquences relatives allant de $29 \%$ à 39,9\%. Par contre, Azzam et Dramaix (2001), Kallel et al (2005) ont rapporté que les infections du site opératoire sont moins fréquentes et souvent dominées par les infections respiratoires. Parmi les patients ayant une IAS, $60 \%$ ont bénéficié d'une exploration microbiologique. Sur les 18 bactéries identifiées, Staphylococcus aureus est prépondérant avec 33,33\%. Ils sont suivis de Escherichia coli et de Pseudomonas aeruginosa qui ont la même fréquence de 22,22\%. Au CHUZ Abomey Calavi / So Ava, Staphylococcus aureus a la plus grande proportion $(44,44$ $\%)$, et Escherichia coli est prépondérant $(33,33 \%)$ dans le $\mathrm{CHZ}$ de Cotonou 5. Cette dominance de Staphylococcus aureus suivi de Escherichia coli puis de Pseudomonas aeruginosa dans notre étude, a été aussi rapportée par Ahoyo et al (2012). Cette fréquence d'isolement de Staphylococcus aureus est due à la nature opportuniste et omniprésente du germe (Nauciel et Vilde 2005). La capacité de survie de Staphylococcus aureus est de plusieurs mois sur des surfaces sèches (Axel et al., 2006). Cette durée lui permet une présence dans l'environnement hospitalier et d'être un pathogène responsable des IAS. Dans le centre hospitalier et universitaire de zone d'Abomey Calavi / So Ava, 44,44\% de Staphylococcus aureus a identifié pendant que Escherichia coli était prépondérant avec $33,33 \%$ dans le centre hospitalier de zone de Cotonou 5. Staphylococcus aureus a été le deuxième micro-organisme plus fréquent du centre hospitalier de zone de Cotonou 5 avec 22,22\% tout comme Acinetobacter spp avec la même proportion. Par contre, Pseudomonas aeruginosa est le deuxième germe qui a une fréquence importante de $33,33 \%$ dans le centre hospitalier et universitaire de zone d'Abomey Calavi / So Ava. Les bactéries multirésistantes aux antibiotiques habituels représentaient $44,44 \%$ et Staphylococcus aureus puis Escherichia coli sont les plus 

sud Benin: Centre Hospitalier Universitaire de Zone d'Abomey-Calavi/Sô-Ava et de Zone de Cotonou 5.

dénombrés. Ces résultats se distinguent de ceux rapportés en 2015 au Centre National Hospitalier et Universitaire Hubert Koutoukou Maga de Cotonou (Bénin) où Klebsiella pneumoniae, Escherichia coli, Acinetobacter spp, Proteus mirabilis et Enterobacter cloacae représentaient $53 \%$ (Ouendo et al. 2015). L'OMS estime que les personnes ayant un SARM (Staphylococcus aureus résistant à la méthicilline) ont une probabilité de $64 \%$ plus élevée de mourir que celles qui ont une forme non résistante de cette infection (OMS, 2016). La résistance microbienne aux antibiotiques existe au Bénin et a été rapportée en 2006 par Ahoyo et al. Celle concernant particulièrement les bactéries Gram-négatives a été perçue par Kunin en 1993. Cette résistance aux antimicrobiens survient naturellement dans le temps, en général à la suite de modifications génétiques mais l'usage abusif ou excessif des antibiotiques accélère le processus (Kunin, 1993 ; OMS 2016). La proportion de bactéries résistantes aux antimicrobiens au Bénin pourrait s'expliquer par la pratique de l'automédication chez les patients et l'utilisation d'antibiotiques de qualité douteuse lors des prises en charges. Dougnon et al (2016) ont perçu, que la qualité des disques d'antibiotiques utilisés pour la réalisation des antibiogrammes pourrait être un

\section{CONCLUSION}

Cette étude rapporte la prévalence des infections associées aux soins dans le Centre Hospitalier Universitaire de Zone d'Abomey-Calavi/Sô-Ava et le Centre Hospitalier de Zone de Cotonou 5. Elle met en évidence l'ampleur du problème de santé, témoin de la qualité des soins dans ces hôpitaux. C'est un indicateur de sensibilisation du personnel soignant. L'identification de bactéries résistantes aux antibiotiques est une autre difficulté dans la prise en charge des patients avec des options thérapeutiques souvent limitées. Le sondage

\section{RÉFÉRENCES}

Abreu AC, Tavares RR, Borges A, Mergulhão F, Simões M., 2013. Current and emergent strategies for disinfection of hospital environments. J Antimicrob Chemother. 68(12):2718-32.

Agut $\mathrm{H}$ 2010. Les virus des infections nosocomiales et professionnelles. Hygiène Hospitalière, volume 3. Sauramps Medical. 79-90.

Ahoyo AT, Baba-Moussa L, Makoutode M, Gbohoun A, Bossou R, Dramane K, Sanni A, Prévost G, 2006. Incidence of meticillin - resistant Staphylococcus aureus in the neonatal care unit of the departmental hospital centre of Zou Collines in Benin. Arch Péd, 13:1391 - 1396. facteur favorisant la résistance des bactéries. Dès lors, il est important de mettre fin à l'automédication et à I'utilisation d'antibiotiques de mauvaise qualité à partir d'une sensibilisation et une information continue de tous les acteurs de santé. Ainsi, une appropriation des recommandations thérapeutiques réactualisées corrigerait l'utilisation inappropriée des antibiotiques dans les pays en développement (Trostle, 1996 ; Joram 2012 ; OMS, 2016). Dans cette étude, le sondage vésical est un facteur de risque d'une infection associée aux soins $\left(O R=2,45 ; \quad C h i-2=42,81\right.$ et $\left.p<10^{-3}\right)$. Ce résultat se rapproche de celui rapporté en 2007 dans une étude réalisée au Centre Hospitalier et Universitaire Hassan II de Fès (Maroc) (El Rhazi et al., 2007). La qualité de l'asepsie avant le sondage vésical pourrait expliquer la survenue de ce facteur de risque. Le nombre réduit de facteurs de risques associés aux IAS dans cette étude en analyse univariée ne nous permet pas efficacement de faire une analyse de régression logistique. L'étude devrait être élargie à d'autres centres hospitaliers du Bénin dans la même période pour être conclusive et sensibiliser d'avantage de professionnels de santé au problème d'IAS et à la résistance bactérienne aux antibiotiques.

vésical est un facteur de risque d'infections associées aux soins dans ces centres hospitaliers. La maîtrise du problème nécessite un programme opérationnel rigoureux en hygiène hospitalière, et une information convenable du personnel soignant sur les infections associées aux soins. L'utilisation efficiente des antibiotiques, et la sensibilisation des patients sur les inconvénients d'une automédication sont également des éléments de prévention des résistances microbiennes aux antibiotiques.

Ahoyo Th A, Bankolé H S, Adéoti F M, Gbohoun A A, Assavèdo S, Amoussou-Guénou, Kindé-Gazard D A, Pittet D, 2014. Prevalence of nosocomial infections and anti-infective therapy in Benin: results of the first nationwide survey in 2012. Antimicrobial Resistance and Infection Control 3:17.

Allegranzi B, Bagheri Nejad S, Combescure C, Graafmans W, Attar H, Donaldson L, Pittet D, 2011. Burden of endemic health-care-associated infection in developing countries: systematic review and meta-analysis. Lancet, 377:228 241. 
Axel K, Ingeborg S, Günter K, 2006. How long do nosocomial pathogens persist on inanimate surfaces? A systematic review. BioMed Central Infectious Diseases 6: 130.

Azzam R et Dramaix M, 2001. A one-day prevalence survey of hospital-acquired infections in Lebanon. Journal of Hospital Infection, 49(1):7478.

Bagheri Nejad S, Allegranzi B, Syed SB, Ellis B, Pittet D, 2011. Health-care-associated infection in Africa: a systematic review. Bull World Health Organ. 89(10):757-65.

Bezzaoucha A, Makhlouf F, Dekkar N, Lamdjadani N, 1994. Prévalence des infections nosocomiales au centre hospitalo-universitaire de Bab $\mathrm{El}$ Oued-Alger. Médecine et Maladies Infectieuses, 24(2):96-101.

Blomberg B, Mwakagile DSM, Urassa WK, Maselle SY, Mashurano M, Digranes A, Stig Harthug S, Langeland N, 2004. Surveillance of antimicrobial resistance at a tertiary hospital in Tanzania. BMC Public Health 4:45.

CDC (Centers for Disease Control and Prevention), 2017. Basic Information Sepsis. https://www.cdc.gov/ sepsis/basic/index.html

Coignard B, Thiolet J, Lacavé L, 2007. Enquête nationale de prévalence des infections nosocomiales. Résultats préliminaires. Institut de veille sanitaire (RAISIN).

Daza R, Gutiérrez J, Piédrola G, 2001. Antibiotic susceptibility of bacterial strains isolated from patients with community-acquired urinary tract infections. Int J Antimicrob Agents., 18(3):211-5.

Decret 2006-087. Politique nationale d'hygiène hospitalière en République du Bénin. 14p

Dougnon TV, Johnson RC, Bankolé HS, Koudjalé B, Hounmanou G, Baba-Moussa L, Boko M, 2016. Évaluation de la performance de trois marques de disques d'antibiotiques vendues au Benin. Health Sci. Dis 17 (4) : 71-78.

Durocher A, 2005. L'infection nosocomiale comme indicateur de (non) qualité des soins : l'exemple de la réanimation (Commentaire). In: Sciences sociales et santé. Volume 23(3) : 59-68.

El Rhazi K, Elfakir S, Berraho M, Tachfouti N, Serhier Z, Kanjaa C, 2007. Prévalence et facteurs de risque des infections nosocomiales au $\mathrm{CHU}$ Hassan II de Fès (Maroc). Revue de Santé de la Méditerranée Orientale, 13(1): 56-63.

Espinasse F, Page B, Cottard-Boulle B, 2010. Risques infectieux associés aux dispositifs médicaux invasifs Revue francophone des laboratoires $426: 51-63$.

Gastmeier P. Gastmeier P, Kampf G, Wischnewski N, Hauer T, Schulgen G, Schumacher M, Daschner F, Rüden H, 1998. Prevalence of nosocomial infections in representative German hospitals. Journal of Hospital Infection, 38:37-49.

Ghazi M, Khanbabaee G, Fallah F, Kazemi B, Mahmoudi S, Navidnia M, Pourakbari B, Bakhshi B, Goudarzi H, 2012. Emergence of Pseudomonas aeruginosa cross-infection in children with cystic fibrosis attending an Iranian referral pediatric center. Iran J Microbiol. 4(3): 124-129.

Goettsch W, van Pelt W, Nagelkerke N, Hendrix MG, Buiting AG, Petit PL, Sabbe LJ, van Griethuysen AJ, de Neeling AJ, 2000. Increasing resistance to fluoroquinolones in $\mathrm{E}$. coli from urinary tract infections in the Netherlands. J Antimicrob Chemother, 46: 223-8.

Haley RW, Culver DH, White JW, Morgan WM, Emori TG, Munn VP, Hooton TM, 1985. The efficacy of infection surveillance and control programs in preventing nosocomial infections in US hospitals American journal of epidemiology, 121:182-205.

Hamza R, 2010. Epidémiologie des infections associées aux soins. Revue Tunisienne d'Infectiologie. 4: 1 -4 .

Joram N, de Saint Blanquat L, Stamm D, Launay E, Gras-Le Guen C. 2012. Healthcare-associated infection prevention in pediatric intensive care units: a review Eur. J. Clin. Microbiol. Infect. Dis.31: 2481-2490.

Kunin C M, 1993. Resistance to antimicrobial drugs. A worldwide calamity. Ann Intern Med, 118: 557 61.

Le Heurt M, Gomila H, Girot S, Pividori I, Rafaoui M J, 2002 Hygiène. Nouveaux cahiers de l'infirmière, 2ème édition, Masson, 6 - 163.

Ministère de la santé, de la jeunesse et des sports DGS/DHOS. 2007. Actualisation de la définition des infections nosocomiales. Comité technique des infections nosocomiales et des infections liées aux soins (CTINILS).

Movahedi Z., Pourakbari B., Mahmoudi S, Sabouni F, Haghi Ashtiani Mt, Hosseinpour Sadeghi R, Mamishi S, 2013. Pseudomonas aeruginosa infection among cystic fibrosis and ICU patients in the referral Children Medical Hospital in Tehran, Iran. J Prev Med Hyg. 2013; 54:24-28.

National Nosocomial Infections Surveillance (NNIS), 2004. System Report, data summary from 
January 1992 through June 2004, issued October 2004. Am J Infect Control, 32:470-485.

Nauciel C, Vilde JL 2009. Bactériologie médicale. In Editions Masson. 2nd edition. Paris, $49-58$.

Organisation des Nations Unies (ONU) 2015. Objectif 3 de Développement Durable. http://www.un.org /sustainabledevelopment/fr/health/. Consulté le 29 Août 2017.

Organisation Mondiale de la Santé (OMS) 2016. L'OMS recommande 29 moyens de mettre fin aux infections en chirurgie et d'éviter les superbactéries. http://www.who.int/mediacentre/ news/releases/2016/recommendations-surgicalinfections/fr. Consulté le 24 Octobre 2017.

Organisation Mondiale de la Santé (OMS) 2016. Résistance aux antimicrobiens. http://www. who.int/mediacentre/factsheets/fs194/fr/ Consulté le 29 Août 2017.

Organisation Mondiale de la Santé (OMS) 2017. Pourquoi un Défi mondial sur les infections nosocomiales. http://www.who.int/gpsc/back ground/fr/. consulté le 24 Octobre 2017

Ouendo EM, Saïzonou J, Dégbey C., Glélé Kakai C., Glélé Y, MAKOUTODE M, 2015. Gestion du risque infectieux associé aux soins et services au Centre National Hospitalier et Universitaire Hubert Koutoukou Maga de Cotonou (Bénin), Int. J. Biol. Chem. Sci. 9(1) : 292-300.

Parneix $P, 2010$. Les infections nosocomiales associées aux soins. Hygiène Hospitalière. Sauramps Medical, 3 ème édition, p37-46.

Pittet D, Harbarth S, Ruef C, Francioli P, Sudre $P$, Petignat C, Trampuz A, Widmer A, 1999. Prevalence and risk factors for nosocomial infections in four university hospitals in Switzerland. Infection control and hospital epidemiology, 20:37-42.

Pividori I et Le Heurt C, 2011. Infectiologie et hygiène. Cahier des sciences infirmières, 2ème édition, Elsevier Masson, 26-120.

Pourakbari B, Ferdosian F, Mahmoudi S, Teymuri M, Sabouni F, Heydari H, Ashtiani M T H, Mamishi $S$, 2012. Increase resistant rates and ESBL production between $\mathrm{E}$. coli isolates causing urinary tract infection in young patients from Iran. Braz J Microbiol 43(2):766-769.

Pourakbari B., Sadr A., Ashtiani M.T.H. 2011. Five-year evaluation of the antimicrobial susceptibility patterns of bacteria causing bloodstream infections in Iran. J Infect Dev Ctries 6 (02):120 125.
Simon F, Kraemer P, De Pina JJ, Demortiere E, Rapp C, 2007. Le risque nosocomial en Afrique intertropicale - Partie 2: les infections des patients. Med Trop, 67:197 - 203.

Soussy C. J, 2010. Principaux micro-organismes responsable: les bactéries. Hygiène Hospitalière. Sauramps Medical, 3: p69-78.

Stone PW, Hedblom EC, Murphy DW, Miller SB, 2005. The economic impact of infection control: Making the business case for increased infection control resources. Am J Infect Control, 33: 5427.

Sydnor ER, et Perl TM 2011. Hospital Epidemiology and Infection Control in acute- care Settings. Clin Microbiol Rev. 24(1): 141-73.

Trostle J, 1996. Inappropriate distribution of medicines by professionals in developing countries. Soc Sci Med, 42(8):1117 - 20. 